\title{
El Archivo Ambrosio Rabanales y la historiografía lingüística chilena e hispanoamericana
}

\author{
Dario Rojas ${ }^{*}$ \\ Tania Avilés \\ Universidad de Chile, Chile
}

La presente nota ${ }^{1}$ tiene el propósito de dar a conocer a la comunidad científica el proceso de constitución del Archivo Ambrosio Rabanales y hacer una breve reseña de sus principales contenidos, destacando los temas que pueden resultar de mayor interés para la historiografía lingüística hispánica del siglo XX.

El archivo personal del profesor Ambrosio Rabanales Ortiz (19172010) fue legado por su familia a la Universidad de Chile tras su muerte ${ }^{2}$.

* Para correspondencia, dirigirse a: Darío Rojas (darioroj@u.uchile.cl), o a Tania Avilés (taviles@ug.uchile.cl), Departamento de Lingüística, Facultad de Filosofía y Humanidades, Universidad de Chile, Av. Capitán Ignacio Carrera Pinto 1025, tercer piso, Ñuñoa, CP 7800284 , Santiago, Chile.

1 Este trabajo se enmarca en el proyecto FONDART 71804 "Rescate del archivo personal de Ambrosio Rabanales: educación y lenguaje en Chile (1936-2010)" (FONDART Regional 2015, Línea Investigación). Una versión preliminar fue presentada en el simposio "Las personas en la Historia de la Lingüística. Archivos, cartas e historia oral en la historiografía lingüística", desarrollado en el marco del XX Congreso de la Asociación Alemana de Hispanistas, Heidelberg (Alemania), 18-22 de marzo de 2015. Agradecemos a Abelardo San Martín por los comentarios y sugerencias que hizo a un primer borrador del presente escrito.

2 El año 2011, sus herederos, en particular su hijo Gabriel Rabanales, se ocuparon de cumplir la voluntad declarada del académico: donar su biblioteca (de la cual formaban parte los materiales que ahora componen el Archivo) a la Facultad de Filosofía y Humanidades 
Pensamos que estos materiales constituyen una veta digna de consideración para la historiografía de los estudios del lenguaje en Chile y Latinoamérica durante el siglo XX, y que su valor historiográfico trasciende la figura del propio Rabanales, pues muestran que este académico actuó varias veces a manera de "vector" para el desarrollo de diversos proyectos e intereses de la lingüística hispánica. Esta es la principal razón que justifica la creación del Archivo, proceso que se encuentra actualmente en curso, en el marco de un proyecto de investigación financiado por el Consejo Nacional de la Cultura y las Artes (FONDART Regional 71804) y patrocinado por la Facultad de Filosofía y Humanidades de la Universidad de $\mathrm{Chile}^{3}$ y por la Academia Chilena de la Lengua (institución que también integró Rabanales). Además del interés historiográfico-lingüístico que reviste, el Archivo podría significar una contribución para el conocimiento de la historia de las instituciones científicas en el país.

\section{AMBROSIO RABANALES COMO "VECTOR" EN LA LINGÜÍSTICA CHILENA E HISPANOAMERICANA}

No pretendemos presentar acá una reseña acabada de la biografía de Rabanales, para cuyo conocimiento pueden consultarse varios estudios anteriores (Contreras 1980-1981; Césped 1980; Empresa Periodística de Chile 1984-86; Jobet 1986; Matus 1980-1981; Sánchez 1998-1999; Valencia 1980-1981, entre otros). Más bien, queremos destacar algunos hechos de su biografía académica que permiten entender de mejor manera el papel de "vector" que le hemos atribuido.

Rabanales fue un connotado gramático (proponente de un modelo funcionalista original; cf. Rabanales 1966) y formador de profesores de lenguaje en Chile, quien, desde 1936 hasta su muerte, formó parte de y

de la Universidad de Chile. Gracias a la gestión del entonces director del Departamento de Lingüística, Abelardo San Martín, y al apoyo de la decana María Eugenia Góngora, dicho trámite fue realizado prontamente. También facilitaron grandemente la gestión Domingo Román, de la Pontificia Universidad Católica, e Hiram Vivanco, de la Universidad de Chile, quienes mediaron en el contacto con la familia Rabanales.

3 Esta institución financió durante el 2014 el trabajo archivístico, sobre todo gracias al decidido apoyo de la entonces directora del Departamento de Lingüística, Susana Serra Sepúlveda, y de la decana de la facultad, María Eugenia Góngora. 
participó activamente en diversas instituciones de relevancia cultural para este país, tales como el Instituto Pedagógico de la Universidad de Chile, la Facultad de Filosofía y Humanidades de esta misma universidad, la Academia Chilena de la Lengua, la Sociedad Chilena de Lingüística y el Círculo Lingüístico de Santiago, entre otras. Cultivó no solo la gramática, sino también la fonología, la ortografía, la lexicografía, la semiología, la estilística, además de interesarse en y promover aproximaciones interdisciplinarias, como los estudios de trastornos del lenguaje. En Sánchez (1998-1999) se encuentra una completa bibliografía de sus trabajos publicados entre 1930 y el 2000 , que dan cuenta de la diversidad de sus intereses intelectuales.

Su formación académica comenzó en 1936, en el Instituto Superior de Humanidades e inmediatamente en el Instituto Pedagógico de la U. de Chile, donde estudió pedagogía en Castellano, Filología Clásica y Filosofía. Siendo estudiante, fue profesor ayudante de la cátedra de Gramática Castellana, y en 1952, tras la muerte del titular Claudio Rosales, Rabanales ocupó su cargo, el que conservaría hasta su muerte. Obtuvo el grado de doctor en la misma Universidad de Chile, en 1954. Durante la década de 1950, también formó parte del equipo de redacción del Boletín de Filología. En 1970 se hizo cargo de la dirección del Instituto de Filología de la Universidad de Chile. En 1991, fue nombrado Miembro de Número de la Academia Chilena de la Lengua y Correspondiente de la Real Academia Española. La Universidad de Chile le otorgó la condición de Profesor Emérito poco antes de su muerte.

Rabanales constituye una figura de gran relevancia en el desarrollo de la lingüística chilena durante el periodo en que estuvo activo, tanto por las figuras de quienes recibió su formación en la Universidad de Chile, como por haber formado a buena parte de la generación actual de maestros de la lingüística chilena. Entre los primeros (los maestros de Rabanales), se cuentan el filólogo Rodolfo Oroz y el gramático Claudio Rosales, a través de quienes se puede atribuir a Rabanales un vínculo de discipulato indirecto con los alemanes Rodolfo Lenz y Federico Hanssen. Si a estos dos últimos personajes se les atribuye el protagonismo de la etapa "fundacional" de la lingüística chilena (1889-1920), a Oroz y Rosales, y luego a Rabanales, les correspondería la etapa de "asentamiento" de la disciplina, en el seno de la Universidad de Chile (Valencia 1993: 138). También, de manera indirecta, a través de sus lecturas, Rabanales tempranamente adoptó ideas centrales de autores como Ferdinand de Saussure, Charles Bally, Karl Vossler, Leo Spitzer, Karl Bühler y André Martinet (la mayoría de ellos a través de las traducciones de Amado Alonso), que se cuentan entre quienes él mismo reconoció como quienes más lo influenciaron intelectualmente. 
Las redes académicas en que participó Rabanales trascienden el contexto chileno, pues también formó parte de numerosas sociedades científicas internacionales. Por ejemplo, desde un comienzo jugó un papel central en la creación de la ALFAL y en el proyecto de estudio coordinado de la "norma culta" del español. Por otra parte, y de esto es testimonio patente el material contenido en el Archivo, Rabanales mantuvo abundante intercambio epistolar con numerosos académicos de universidades extranjeras, de las cuales también varias veces fue profesor visitante (principalmente en Alemania: Augsburgo, Bonn, Colonia, Heidelberg y Leipzig; y en otras partes del mundo: Pekín, Bucarest, Nimega, Utrecht, Basilea, Coimbra, Autónoma de Madrid, Barcelona, Nacional Autónoma de México, Central de Quito, Nacional de San Juan, etc.). Los materiales depositados en el Archivo Ambrosio Rabanales dan cuenta de todas estas líneas de su trayectoria, y también pueden encontrarse en ellos trazos de las historias académicas extranjeras que confluyen en los intercambios señalados.

\section{EL ARCHIVO AMBROSIO RABANALES}

Los materiales de Rabanales legados a la Universidad de Chile han sido objeto de un trabajo técnico archivístico que comenzó el 2014, y que será continuado el 2015 gracias a un proyecto de investigación financiado por el Consejo Nacional de la Cultura y las Artes de Chile (proyecto FONDART 71804), dirigido y ejecutado por los autores de esta comunicación, con la asistencia de una conservadora y restauradora profesional (Carolina González Bravo) y con el apoyo investigativo de Susana Serra, del Departamento de Lingüística de la Universidad de Chile.

El Archivo se encuentra constituido por dos secciones claramente definidas, según la tipología de los materiales: fichas (92 registros y 8488 piezas en total) y documentos (2346 registros y 5770 piezas inventariadas hasta el momento). Todos estos materiales han sido inventariados en una planilla de Excel, en consideración de los campos descriptivos relativos al área de identificación de las unidades documentales (cf. ISAD- G y Manual Marc/Sinfonia para el ingreso de Archivos y/o Colecciones de Manuscritos, SISIB, Universidad de Chile).

Uno de nuestros propósitos ha sido intentar conservar el orden original del fondo, en consideración de los principios de procedencia y orden primitivo (ISAD-G). Estos documentos fueron producidos y acumulados por Ambrosio Rabanales en el desarrollo de su propia actividad profesional 
y fueron conservados en su hogar. Suponemos que el orden en que fueron entregados los materiales a la universidad, junto con su biblioteca personal ${ }^{4}$, es el que poseían al momento de salir de la vivienda del profesor Rabanales. En este mismo orden fueron trasladados al Departamento de Lingüística de la Universidad de Chile. La organización de ellos que estamos realizando no adulterará dicho orden ni su clasificación (cf. Fugueras 2003, cit. en Eslava 2008).

Los documentos que conforman el Archivo Personal de Ambrosio Rabanales fueron almacenados inicialmente en tres cajas de cartón. En la primera caja, habían 8 archivadores antiguos, de un color verdoso, y con una etiqueta de identificación "tópica" en el lomo: L, Haensch, Lope I, Lope II, Polo, Círculo-Biblioteca del Congreso XI, Programas Bibliografías Materiales, SOCHIL. Estos archivadores contienen correspondencia, documentación administrativa y material docente, entendiendo por esto último pruebas, bibliografías y programas de estudio. Físicamente, se trata de hojas de oficio o carta, manuscritas o mecanografiadas. Cabe señalar que entre los archivadores se encuentra uno titulado " $L$ ", que corresponde a un conjunto de documentos pertenecientes a Lidia Contreras (1919-1992), quien fue esposa de Rabanales y que, igualmente, colaboró como intelectual en el ámbito de la lingüística general y la gramática española en la Universidad de Chile. Aun cuando, en estricto rigor, no debiésemos considerar este conjunto archivístico como parte del archivo del profesor Rabanales, será rescatado en honor al estrecho vínculo académico que Contreras sostuvo con quien fue además su maestro.

En la segunda caja se encontraban diversas carpetas y algunos materiales sueltos. Estas carpetas, al igual que los archivadores, contienen correspondencia, documentación administrativa y material docente. Físicamente, se trata de hojas de oficio o carta, manuscritas o mecanografiadas en su mayoría y recortes de prensa.

Por último, en cuanto al fichero, almacenado en la tercera caja, cabe señalar que las fichas se encontraban agrupadas temáticamente por el productor y atadas con pita de algodón o plástico, otras con clips metálicos, corchetes y elásticos que, con el tiempo, se vencieron. Aunque nuestro equipo no ha abordado todavía el estudio exhaustivo de estas fichas, parece tratarse fundamentalmente de fichas bibliográficas, apuntes de estudio y fichas lexicográficas.

\footnotetext{
4 Hoy en día, la biblioteca del profesor Ambrosio Rabanales se encuentra depositada en la Biblioteca Central de la Facultad de Filosofía y Humanidades de la Universidad de Chile. Los libros que la componían están catalogados bajo el nombre de Colección Ambrosio Rabanales.
} 
En consideración de orden primitivo del archivo personal en cuestión, creemos que la estructura general de este debe considerar una perspectiva temática de acceso a los materiales. De esta manera, la organización preliminar del archivo considera los siguientes tópicos (que presentamos por orden alfabético según el descriptor que hemos asignado a cada uno):

1) Círculo Lingüístico de Santiago: Rabanales fue protagonista de la fundación del "Círculo Lingüístico de Santiago", activo al menos entre 1958 y 1971, cuya preocupación principal fue difundir en el contexto académico chileno los avances de esta ciencia en Europa, lo cual, en ese momento, significaba básicamente el estructuralismo europeo. Rabanales, de hecho, fue uno de los primeros grandes defensores y difusores de la doctrina estructuralista europea en el medio académico chileno, y él mismo reconoció públicamente su afinidad con teóricos como Eugenio Coseriu, con quien mantuvo una larga relación académica.

El conjunto de documentos al que nos referimos corresponde al archivo bibliográfico del Círculo, que fue pensado como apoyo para la docencia universitaria. Consta de dos series: Traducciones y Reproducciones. Se trata de artículos de investigación lingüística numerados, de los cuales se conservan, en el Archivo Rabanales, del n..$^{\circ} 1$ al 14, del 16 al 25 y el 27. Destacan traducciones de trabajos de Louis Hjelmslev (Acta Grammatica Editorial, trad. por Rabanales), Serge Karcesvski (La dualidad asimétrica del signo lingüístico, trad. por Lidia Contreras), Viggo Brøndal (El sistema de la gramática, trad. por Nelson Cartagena), W. Borgeaud, W. Bröcker y J. Lohmann (La naturaleza del signo, trad. por Adalberto Salas), George L. Trager (El campo de la lingüística, trad. por Rabanales), Roman Jakobson (Las funciones del lenguaje, trad. por Lidia Contreras) y Noam Chomsky (Situación actual de la lingüistica: tendencias actuales, trad. por Lidia Contreras), entre otros. También incluye trabajos del propio Rabanales (Las funciones gramaticales, Métodos probatorios en gramática científica, etc.) y de Lidia Contreras (Significados y funciones del 'se', Oraciones y clausulas interrogativas con "si", etc.), así como del maestro de Rabanales, Claudio Rosales.

2) Comisión sobre el idioma oficial de Chile: En el año 1993, el Ministerio de Educación de Chile solicitó a la Academia Chilena de la Lengua (y a representantes de otras instituciones como la Universidad de Chile, la Universidad Metropolitana de Ciencias de la Educación, el Centro de Perfeccionamiento, Experimentación e Investigación Pedagógicas y la División de Educación General) la constitución de una comisión de trabajo, coordinada por Ambrosio Rabanales, cuyos objetivos serían determinar 
una denominación oficial para la lengua dominante en Chile ("¿castellano o español?") y establecer la composición del abecedario de esta lengua (se proponía incluir como letras autónomas los dígrafos $c h, l l$ y $r r$ ).

Tras la revisión bibliográfica y discusiones en las reuniones de la comisión, se propone que el nombre oficial del idioma en Chile sea español y que el abecedario español quede constituido por 30 letras. El conjunto documental agrupado bajo este rótulo contempla bibliografía, actas de reuniones y recortes de prensa relativos a esta iniciativa, de interés para la historia de la planificación lingüística en Chile.

3) Günther Haensch: La relación académica que vinculó a Rabanales con el lingüista alemán Günther Haensch surge en el marco del proyecto Nuevo Diccionario de Americanismos, dirigido y financiado por la Universidad de Augsburgo. Dentro de la serie de diccionarios por elaborar, se consideraba un Nuevo diccionario de chilenismos, cuya redacción estaría a cargo de un equipo conformado por Rabanales, Lidia Contreras, Alfredo Matus y Alba Valencia. Este diccionario nunca llegó a publicarse, de modo que los documentos conservados en el Archivo Rabanales constituyen la parte más importante de las escasas huellas que hay de tal proyecto. Es importante tener en cuenta que, en el momento en que se gesta este proyecto, aún no se publicaba el Diccionario ejemplificado de chilenismos de Félix Morales Pettorino y su equipo (de 1983), de modo que el proyecto de Augsburgo habría sido, de haberse concretado rápidamente, el primer diccionario científico del español de Chile.

Entre los documentos contenidos en esta sección del Archivo, destaca la abundante correspondencia entre Haensch y el equipo de trabajo chileno (cartas datadas entre 1976 y 1997), informes de avance de cada uno de ellos, informes sobre la visita de Haensch a Chile y de las visitas de Rabanales y Matus en calidad de Gastprofessor. Entre los documentos administrativos, son relevantes el convenio de cooperación entre la Universidad de Chile y la Universidad de Augsburgo (1979) y algunos materiales de trabajo como bibliografía y apuntes redactados por Haensch, además de las instrucciones del Nuevo Diccionario de Americanismos para la recogida de materiales, redacción de fichas y contestación de cuestionarios.

4) José Polo: Este conjunto documental contiene correspondencia sostenida entre Ambrosio Rabanales y el lingüista español José Polo a propósito de dos proyectos editoriales del gramático chileno, que Polo apoyó y ayudó a concretar. Uno de estos proyectos fue la edición y publicación de los Métodos probatorios en gramática científica en Editorial Istmo; el otro, la publicación de una antología titulada Ortografía y grafémica (Editorial 
Visor), obra póstuma en homenaje a Lidia Contreras. Además, destacan los intercambios bibliográficos y de noticias relativas a sus ámbitos de interés compartidos entre ambos intelectuales. En específico, en un par de cartas José Polo advierte a Rabanales de algunas investigaciones recientemente publicadas, como Las categorías gramaticales (Síntesis, 1989) de Ignacio Bosque, que podían "afectar" el carácter de novedad de los Métodos probatorios de Rabanales.

5) Juan Manuel Lope Blanch: Contiene documentación administrativa y correspondencia entre Rabanales y el lingüista mexicano Juan Manuel Lope Blanch, a propósito de la Asociación de Lingüística y Filología de la América Latina (ALFAL) y el Proyecto de Estudio de la norma lingüistica culta de las principales ciudades de Iberoamérica y de la Península Ibérica. Con respecto a la ALFAL, la documentación es fundamentalmente administrativa (Rabanales fue secretario de la institución desde su fundación hasta 1978), lo cual se refleja claramente en las palabras del mismo Lope Blanch (en carta dirigida a Edwin Figueroa): "En cuanto a la información precisa que necesitas sobre ALFAL, creo que sólo Rabanales puede proporcionártela, pues él, como Secretario de la Asociación, es el único que tiene el archivo general". (LOPEI_369). Destacan también documentos relativos a la organización de los congresos internacionales de ALFAL entre los años 1975 y 1987 y las vinculaciones de la sociedad con, por ejemplo, el Programa Interamericano de Lingüística y Enseñanza de Idiomas.

Entre los proyectos de aquellos años a los que se hace referencia en los documentos, se encuentran la redacción de la Historia de la ALFAL, la publicación de los Cuadernos de Lingüística por el Instituto Caro y Cuervo, el Boletín informativo de la Sociedad y las Actas del congreso de Viña del Mar, texto fundacional de la Sociedad correspondiente a la primera reunión latinoamericana de lingüística y filología realizada en el año 1964, en Viña del Mar, Chile ${ }^{5}$. En cuanto al Proyecto del Estudio de la Norma Culta, la proporción de documentos es mucho menor, y destaca la correspondencia relativa al proceso de publicación del Léxico del habla culta de Santiago, en cooperación con la Universidad Nacional Autónoma de México, circulares e informes de avance de las distintas ciudades participantes del proyecto (sobre todo de Santiago de Chile, por haber dirigido Rabanales el proyecto en el país).

$5 \quad$ Fuente: Actas de la primera reunión latinoamericana de lingüistica y filología (enero de 1964). Bogotá: Instituto Caro y Cuervo, 1973. 
6) Lidia Contreras: La figura de Lidia Contreras (1919-1992) es muy relevante para comprender íntegramente la de Ambrosio Rabanales, pues además de colaboradores y académicos del actual Departamento de Lingüística de la Universidad de Chile, fueron esposos. En palabras de Sánchez, quien a su vez cita a Rabanales, "En su vida fue todo para él, 'una muy buena compañera, una excelente colaboradora'. Tenían una gran afinidad, desde todo punto de vista, lo cual es fundamental para una buena convivencia" (Sánchez 1998-1999: 43). A través de un amplio conjunto epistolar puede observarse la constante actividad académica, en colaboración con Rabanales, que desarrolló Contreras: participación en sociedades, congresos nacionales e internacionales, proyectos de investigación y publicaciones académicas. No obstante, los archivos que se conservan de ella dejan ver también sus labores como destacada docente, por ejemplo, correspondencia con estudiantes y académicos chilenos y extranjeros, interesados en sus publicaciones y en las observaciones críticas que ella podía esbozar al evaluar sus trabajos inéditos. Los trabajos más comentados de Lidia Contreras fueron Significados y funciones del 'se' y El periodo comparativo hipotético con 'si'. También se puede observar el proceso formativo de Contreras en el marco del Seminario Románico de la Universidad de Bonn, dirigido por Harri Meier. Se suman a estos documentos aquellos vinculados a su elección como Miembro de Número de la Academia Chilena de la Lengua (1990) y el otorgamiento del premio "Consejo de Rectores en el Bicentenario del Nacimiento de don Andrés Bello" (1981).

7) Mary Ritchie Key: Esta sección contiene correspondencia (personal y oficial) entre la antropóloga estadounidense Mary Ritchie Key y Ambrosio Rabanales, enviada entre 1975 y 1995, a propósito del proyecto Intercontinental Dictionary Series (IDS), dirigido en su etapa inicial por la académica de la Universidad de Irvine, California (y hoy a cargo de Bernard Comrie). Ritchie Key desarrolló e impulsó la investigación de lenguas indígenas de México y América del Sur, favoreciendo los estudios comparativos y los equipos transculturales. En este contexto, toma contacto con Rabanales para invitarlo a colaborar como editor asociado en el primer volumen del IDS, dedicado a las lenguas indígenas de América del Sur, pero que pretendía "establecer un repertorio básico de datos léxicos representativos de las lenguas de todos los continentes" (MRK_003).

8) Programas, bibliografía y materiales: Tal como lo indica el nombre de la serie, este conjunto de archivos lo conforman tres tipos documentales diferentes, de interés sobre todo para los estudios historiográficos acerca de la enseñanza de la lingüística en la universidad chilena, específicamente, en 
el Departamento de Lingüística de la Universidad de Chile. En primer lugar, y con la mayor presencia cuantitativa, destacan los programas de cursos de la licenciatura y la maestría en Lingüística, mención Lengua Española, fechados entre 1958 y 1999. Entre los cursos dictados por Ambrosio Rabanales se encuentran Dialectología Española, Estilística Lingüística, Fonología Española, Gramática Española, Ortografía y Grafemática, Gramática Española Sincrónica I, II, III y IV, Gramática Española Fundamental, Lingüística Estructural, Sociolingüística, Morfosintaxis III y IV, entre muchos otros. En segundo lugar, se encuentran bibliografías básicas de temas específicos como Fonética y Fonología General, Historia de la Lingüística, Lexicología, Diccionarios Lingüísticos, Gramática Generativa y Transformativa, El Español de América, Morfosintaxis Española, Sociolingüística, Lingüística Histórica Hispánica, Lingüística Románica, etc. Por último, esta sección contiene pruebas de cátedra, pruebas de compensación, exámenes semestrales y controles de lectura.

9) Sociedad Chilena de Lingüística (SOCHIL): Se trata principalmente de documentación administrativa de esta sociedad, fechada entre los años 1970 y 1998, y correspondencia entre Ambrosio Rabanales y varios lingüistas chilenos de la época. Rabanales presidió SOCHIL entre 1971 y 1983. Destacamos especialmente aquella documentación de carácter fundacional relativa a la realización del Primer y Segundo Seminario de Investigación y Enseñanza de la Lingüística, pues en este último evento se fundó la Sociedad Chilena de Lingüística (septiembre de 1971). Aquí hay circulares que anuncian la creación de la sociedad, la nómina de socios fundadores, los estatutos, etc. Rabanales desempeñó un papel fundamental en estas actividades, ya que presidió hasta 1981 la Comisión de Continuidad, que desde 1970 había promovido la creación de la sociedad. El grueso de la documentación, por tanto, refleja este período administrativo del directorio de SOCHIL.

Entre las comisiones de trabajo creadas, destaca la información sobre la Comisión de Planes y Programas, dedicada a elaborar proyectos de planes y programas de estudio para la obtención de grados académicos en lingüística en Chile. Asimismo, SOCHIL promovió la creación de departamentos autónomos de lingüística en las universidades nacionales que por esa fecha (1971) no tenían uno.

En el marco de su presidencia de SOCHIL, Rabanales organizó la venida a Chile, como invitados de la sociedad, de André Martinet y su esposa, Günther Haensch y Juan M. Lope Blanch; vinculó a la sociedad chilena con la ALFAL y patrocinó actividades académicas interdisciplinarias y pioneras 
en el país, tales como un curso sobre afasias dictado por el doctor Archibaldo Donoso, de la Facultad de Medicina de la Universidad de Chile.

10) Varia: Hemos denominado Varia a un conjunto de documentos que corresponde a un cúmulo de materiales relativos a diversos temas y actividades en las que participó el profesor Rabanales. No descartamos que, durante nuestro trabajo en el periodo 2015, podamos generar otra sección a partir de algún grupo de documentos agrupados por ahora bajo este rótulo.

Entre estos materiales se encuentran circulares y programas de congresos; actas de sesiones ordinarias y extraordinarias de la Academia Chilena de la Lengua, mientras Rabanales fue Miembro de Número de la Institución; materiales del Seminario Permanente de Estudios Semiológicos, dirigido por Rabanales; documentación relativa a las asesorías y servicios que Rabanales prestó a la enciclopedia Encarta y a la Universidad Gabriela Mistral (Chile), etc. Destaca en este conjunto un cuaderno en que Rabanales coleccionó recortes de prensa en los que se hacía referencia a su persona u obras, que abarcan desde su temprana juventud (por ejemplo, su participación en concursos de poseía mientras era un estudiante escolar) hasta su madurez profesional. Junto con esto, sobresale un ejemplar de la primera edición de sus Métodos probatorios en gramática científica, anotado copiosamente por el mismo Rabanales.

\section{CONSIDERACIONES FINALES}

Como puede apreciarse, el Archivo Ambrosio Rabanales contiene información que interesa no solo en relación con el propio Rabanales, sino también en relación con las múltiples redes académicas, institucionales y personales, en que estuvo inserto, redes que se proyectan tanto dentro del continente americano como hacia el resto del mundo. Creemos que la historiografía lingüística hispánica, específicamente la que tiene por objeto el contexto chileno y latinoamericano de los tres últimos cuartos del siglo XX, podría sacar buen provecho de estos materiales. Nuestra comunicación es una invitación a que los investigadores consulten, una vez concluido el proceso de creación y digitalización del Archivo (posiblemente el 2016), los materiales en él contenidos. Garantizamos la mejor disposición para recibir las solicitudes de acceso al Archivo, dentro de lo que nuestras posibilidades materiales nos permitan. 
De manera similar a casos emblemáticos y bien conocidos como los de Hugo Schuchardt o Rodolfo Lenz, la documentación legada por Rabanales da cuenta de que los testimonios personales de los propios lingüistas ofrecen renovadas perspectivas para la historiografía lingüística. El Archivo Ambrosio Rabanales, además, presenta una particularidad: en el fondo, es un acervo documental creado y ordenado por el propio autor (la tarea se nos ha hecho fácil, en este sentido), revelador de qué era considerado por él mismo como relevante en su trayectoria académica. En suma, hasta cierto punto, este Archivo tiene carácter autobiográfico: una autobiografía del propio Rabanales y un relato personal del desarrollo de la lingüística chilena e hispanoamericana en el siglo XX, interesante tanto por lo que dice como por lo que calla.

\section{REFERENCIAS BIBLIOGRÁFICAS}

CÉsped, Irma. 1980. Rabanales y la poesía. Revista Chilena de Literatura 15: 99-105.

Consejo Internacional de Archivos. 1999. ISAD(G): Norma Internacional General de Descripción Archivística. Adaptada por el Comité de Normas de Descripción. Estocolmo, Suecia.

CONTRERAS, Lidia. 1980-1981. Bibliografía cronológica de Ambrosio Rabanales: 1930- 1979. Boletín de Filología 31(1): 61-90.

Empresa Periodística de Chile (eds.). 1984-86. Ambrosio Rabanales. En Diccionario biográfico de Chile. 18. ${ }^{a}$ ed. Santiago: Editorial Universitaria.

Eslava Ochoa, Esther. 2008. Clasificación del Archivo Personal de Francisco Navarro Villoslada. Comunicación presentada en el VIII Congreso de ANABAD, Memoria y tecnología, Madrid.

Jobet, JoRge. 1986. Ambrosio Rabanales, una vocación por el lenguaje. Revista Chilena de Humanidades 8: 69-89.

Matus, Alfredo. 1980-1981. Proemio para un homenaje. Boletín de Filología 31(1): 15-19.

Rabanales, Ambrosio. 1966. Las funciones gramaticales. Boletín de Filología 18: 235-276.

SÁnchez, Gilberto. 1998-1999. El Dr. Ambrosio Rabanales Ortiz. Boletín de Filología 37(1): $19-45$.

Valencia, Alba. 1980-1981. Ambrosio Rabanales. Semblanza de una vocación ejemplar. Boletín de Filología 31(1): 21-60.

1993. El legado de tres maestros: Lenz, Oroz y Rosales. Revista de Lingüistica Teórica y Aplicada 31: 137-162. 